\title{
Synthesis, characterization and photocatalytic activity of porous manganese oxide doped titania for toluene decomposition
}

\author{
R. Jothiramalingam, M.K. Wang * \\ Department of Agricultural Chemistry, National Taiwan University, Taipei 106, Taiwan
}

Received 1 November 2006; received in revised form 11 January 2007; accepted 12 January 2007

Available online 23 January 2007

\begin{abstract}
The present study describes the photocatalytic degradation of toluene in gas phase on different porous manganese oxide doped titanium dioxide. As synthesized birnessite and cryptomelane type porous manganese oxide were doped with titania and tested for photocatalytic decomposition of toluene in gas phase. The effects of the inlet concentration of toluene, flow rate (retention time) were examined and the relative humidity was maintained constantly. Thermal and textural characterization of manganese oxide doped titania materials were characterized by X-ray diffraction (XRD), thermogravemetry (TG), BET and TEM-EDAX studies. The aim of the present study is to synthesize the porous manganese oxide doped titania and to study its photocatalytic activity for toluene degradation in gas phase. Cryptomelane doped titania catalyst prepared in water medium [K-OMS-2 (W)] is shown the good toluene degradation with lower catalysts loading compared to commercial bulk titania in annular type photo reactor. The higher photocatalytic activity due to various factors such as catalyst preparation method, experimental conditions, catalyst loading, surface area, etc. In the present study manganese oxide OMS doped titania materials prepared by both aqueous and non-aqueous medium, aqueous medium prepared catalyst shows the good efficiency due to the presence of $\mathrm{OH}$ bonded groups on the surface of catalyst. The linear forms of different kinetic equations were applied to the adsorption data and their goodness of fit was evaluated based on the $R^{2}$ and standard error. The goodness to the linear fit was observed for Elovich model with high $R^{2}$ ( $\geqq 0.9477$ ) value.
\end{abstract}

(C) 2007 Elsevier B.V. All rights reserved.

Keywords: Birnessite; Cryptomelane; Manganese oxide; OMS; Titanium dioxide; Toluene

\section{Introduction}

Volatile organic compounds (VOCs) are widely used in (and produced by) both industrial and domestic activities [1]. This extensive use results in their occurrence in aquatic, soil and atmosphere environments [2]. Many VOCs are toxic and some are considered to be carcinogenic, mutagenic, or teratogenic [3]. The pollution of indoor volatile organic compounds (VOCs) has been increasingly concerned in recent years. Heterogeneous photocatalysis is regarded as a promising technique for purifying indoor VOCs. A large quantity of work has been devoted in this field in the past decade [4-6]. The $\mathrm{TiO}_{2}$-sensitized photo degradation of organic compounds has been proposed as an alternative advanced oxidation process (AOP) for the decontam-

\footnotetext{
* Corresponding author. Tel.: +8862 3366 4808/2118.

E-mail address: mkwang@ntu.edu.tw (M.K. Wang).
}

ination of water and air. The AOP is initiated from the generation of hole-electron pairs on the semiconductor, absorbing the ultraviolet (UV) light with energy equal to or higher than the band gap energy $\left(E_{\mathrm{g}}\right)$ of semiconductor. Electrons and holes are photogenerated in the bulk of the semiconductor, and move to the particle surface, electrons reduce an electron acceptor such as molecular oxygen and holes can oxidize electron donors including adsorbed water or hydroxide anion to yield hydroxyl radicals.

The common VOCs such as halogenated hydrocarbons, ketones, alcohols and aromatic compounds have been widely used in many industries and are often found in the emission flow [7]. The sol-gel method synthesized anatase-rutile phase transformation of $\mathrm{TiO}_{2}$ containing various amount of $\mathrm{Mn}^{2+}$ ions have been recently reported and they studied the effects of manganese(II) dopants on acceleration of rutile phase formation [8]. Manganese oxides have been reported to be among the most efficient transition-metal oxide catalysts for catalytic disposal of pollutants $[9,10]$. It has been widely used in selective catalytic 
reduction of $\mathrm{NO}_{x}$ with $\mathrm{NH}_{3}$ [11,12], $\mathrm{CO}$ and VOC oxidation $[13,14]$. Heterogeneous photocatalysis in the presence of semiconductor oxides is a fast growing field of basic and applied research, especially for the case of the oxidation of organic pollutants in water or in air [15-17]. In particular, photocatalytic oxidation offers various advantages compared with traditional treatment methods because the photoreactions occur at $25^{\circ} \mathrm{C}$ and atmospheric pressure, under radiation of the near-UV region and at measurable rates [18] until to very low concentration levels. Toluene is a widespread reagent used for the preparation of many compounds, such as benzaldehyde, benzyl alcohol, benzoic acid, chloro derivatives, etc. Consequently, it can be found in many industrial waste effluents. Toluene is a very noxious organic compound and many strategies have been identified to reduce its presence in the environment. Heterogeneous photocatalytic method has been tested for toluene abatement and previous papers report the partial or complete oxidation of toluene both in gas-solid [19-23] and in liquid-solid systems [24-28]. As to concern the gas-solid regime, Ibusuki and Takeuchi [19] carried out the complete photo-oxidation of toluene on $\mathrm{TiO}_{2}$ at $303 \mathrm{~K}$. They found that the presence of water vapour was beneficial in order to achieve the almost complete mineralisation of toluene and benzaldehyde having been detected only in very small amounts. However, the product distribution and the catalyst stability strongly depend on the nature of the catalyst and the experimental conditions. Furthermore, Obee and Brown [20] studied the influence of the competitive adsorption of water and toluene vapours on the photo-oxidation rate.

Luo and Ollis [21] and Einaga et al. [22], studying the toluene degradation in humidified air, report that the influence of water is complex in the sense that an optimum water concentration was found when the maximum reactivity was observed. In any case no significant amounts of intermediate species were detected. Recently the selective photo-oxidation of gaseous toluene to benzaldehyde on $\mathrm{TiO}_{2}$ powders has been reported as an effective method to transform this compound to a valuable chemical compound [23]. Moreover, Cao et al. [24] reported the photocatalytic oxidation of toluene by using nanostructured $\mathrm{TiO}_{2}$ catalysts. They found a severe deactivation of $\mathrm{TiO}_{2}$ due to the accumulation of partially oxidized intermediates. The complete recovery of catalytic activity required a thermal treatment of the photocatalysts at a temperature above $693 \mathrm{~K}$. They reported the formation of cresols, benzaldehyde and benzyl alcohol depending on the $\mathrm{pH}$ of the solution and on the used semiconductor. The formation of benzaldehyde was also confirmed by Navio et al. [25], in their experiment acetonitrile was used as solvent and investigated the influences in the presence of water in product distribution. Recently the use of surfactant to enhance the photocatalytic reaction rate for toluene degradation has also been reported [26,27]. The presence of surfactant or humic acids was found beneficial for degrading various substrates and a sequestration effect has been hypothesized for explaining this effect [27]. The main aims of the present study were to synthesize the porous manganese oxide doped titania and to find out the more active manganese oxide OMS doped titanium dioxide for photocatalytic activity for toluene degradation in gas phase at lower catalyst loading.

\section{Materials and methods}

The commercially available poly crystalline titanium dioxide (Nacalai Tesque, Japan) was used in the present work. To identify the crystalline phases of the samples, Rigaku Geigerflex X-ray diffractometer was used. The $\mathrm{Cu} \mathrm{K} \alpha$ radiation was generated at $40 \mathrm{kV}$ and $30 \mathrm{~mA}$. Samples were scanned from $5^{\circ}$ to $85^{\circ}(2 \theta)$ at a rate of $5 \mathrm{~s}$ per step for all porous manganese oxide doped titania samples. Brunauer-Emmett-Teller (BET) surface area of all samples at $77 \mathrm{~K}$ were obtained using Quantachrome (Model 1987) instrument. All the samples were degassed at $573 \mathrm{~K}$ for $12 \mathrm{~h}$. The instrument used for nitrogen adsorption analysis is NOVA 2000 from Quantachrome Corporation. The morphology and elemental analysis of the catalyst (average of five data points at different locations of the solid) were measured by energy dispersive X-ray spectroscopy. Transmission electron micrographs (TEM) were examined using Philips CM12/STEM, Scientific and Analytical Equipment.

\subsection{Synthesis of potassium birnessite and cryptomelane (porous manganese oxides)}

Potassium birnessite (K-OL-1 type manganese oxide) was synthesized by the oxidation of $0.50 \mathrm{M}$ solution of manganese(II)acetate in strong alkaline medium. Hydrous $\mathrm{Mn}(\mathrm{OH})_{2}$ suspension was obtained by the drop wise addition of $70 \mathrm{~mL}$ of $5.0 \mathrm{M} \mathrm{KOH}$ solution to $40 \mathrm{~mL}$ of $0.50 \mathrm{M}$ manganese(II) acetate. As synthesized manganese oxide suspension was then oxidized by $50 \mathrm{~mL}$ of $0.1 \mathrm{M}$ potassium permanganate solution $\left(\mathrm{MnO}_{4}{ }^{-} / \mathrm{Mn}^{2+}=0.25\right)$ followed by ageing the precipitated suspension for $4-5$ days aging. In general molar ratio between $\mathrm{MnO}_{4}{ }^{-} / \mathrm{Mn}^{2+}=0.2-0.4$ is the favour for the formation of layered structured birnessite. After 4-5 days aging, the suspension was filtered, washed 4-5 times with double distilled water (DDW) and dried at $298 \mathrm{~K}$. Cryptomelane type manganese oxide K-OMS-2 material was synthesized by oxidation of manganese(II) sulfate by potassium permanganate in strong acidic medium under suitable reflux conditions. Hundred millilitres of $0.4 \mathrm{M}$ solution of potassium permanganate was mixed with $31.0 \mathrm{~mL}$ of $1.7 \mathrm{M}$ solution of manganese(II) sulfate hydrate solution. The molar ratio between potassium permanganate and manganese(II) sulfate was fixed at 0.76 $\left(\mathrm{MnO}_{4}{ }^{-} / \mathrm{Mn}^{2+}=0.76\right)$ for preparing cryptomelane K-OMS-2 material in acidic medium. After the addition of an aqueous manganese(II) sulfate solution to the potassium permanganate solution, $15-20 \mathrm{~mL}$ of concentrated nitric acid $(6.0 \mathrm{M})$ was added dropwise to maintain acidic medium and the mixed solution was then stirred at $363 \mathrm{~K}$ for $24 \mathrm{~h}$. The synthesized material was washed with DDW and then the solid brown-black K-OMS-2 material was dried at $383 \mathrm{~K}$ for $8 \mathrm{~h}$ [28].

\subsection{Synthesis of porous manganese oxides doped titania catalysts}

Different weight percentage loading of manganese oxide OMS-titania catalysts was prepared by suitable condition. In 
the present study, 5 and $10 \mathrm{wt} \%$ potassium birnessite (K-OL-1) doped titania ( $2.0 \mathrm{~g}$ commercial titanium dioxide) was prepared in non-aqueous medium, 5, 10 and $15 \mathrm{wt} \%$ cryptomelane (KOMS-2) doped titania and $5 \mathrm{wt} \% \mathrm{MnSO}_{4}$ doped titania (5 wt $\%$ manganese sulfate stirred with titania in acetone) has also been synthesized, which is designated as $5 \mathrm{wt} \% \mathrm{MnSO}_{4}$. The appropriate amount $(2.0 \mathrm{~g})$ of titanium dioxide mixed with suitable amount of K-OL-1 and K-OMS-2 material in $20 \mathrm{~mL}$ acetone and only one sample prepared in water medium such as $2.0 \mathrm{~g}$ of titanium dioxide stirred with $20 \mathrm{~mL}$ of water and stirred with K-OMS-2 (10 wt \%), which is designated as $10 \mathrm{wt} \% \mathrm{~K}-\mathrm{OMS}-2$ (W). After stirred the mixed titanium dioxide and manganese oxide OMS material were subjected to calcined at $723 \mathrm{~K}$ for $4 \mathrm{~h}$. As synthesized manganese oxide doped titania materials are used for photocatalytic experiments.

\subsection{Preparation of manganese oxide OMS-titania coated plastic nylon sheet}

Appropriate amount of porous manganese oxide doped titania (1.0-2.0 g range) mixed with poly acetonitrile (PAN) in alcoholic medium, were poly acetonitrile is act as binder to stick the materials strongly on the nylon sheet (i.e., cheese paper type) under this experimental conditions. The synthesized viscous solution finely coated on plastic nylon sheet and dried in $303 \mathrm{~K}$. The synthesized sheet kept inside the photocatalytic reactor in the rolled form.

\subsection{Experimental setup and analyses procedure}

The saturated toluene gas was prepared by passing air through a saturator containing liquid toluene. The humidified air stream was generated by bubbling air through a thermostated glass bottle containing deionized water. Then the saturated toluene gas was mixed and diluted with humidified air stream at the gas mixer (mixing chamber). The obtained toluene gas stream entered into the photoreactor with constant flow rates $5.0 \mathrm{~L} / \mathrm{min}$, inlet toluene concentration range between 70 and $80 \mathrm{ppmv}$ and relative humidity (RH) in the range of $20-60 \%$. Feed toluene concentrations and relative humidity were set by varying the ratio of gas flow rates and/or varying the saturator temperature.

The outside of the photoreactor was a cooling water sleeve to maintain stable reaction temperature (293-295 K). The $85 \mathrm{~W}$ UV lamp was used to illumination with maximum light intensity output of $254 \mathrm{~nm}$. The lamp was fixed at the center of the photoreactor without any quartz tube protection. The manganese oxide doped titania material coated on nylon sheet (length $8 \mathrm{~cm}$, height $10 \mathrm{~cm}$ and thickness $0.18 \mathrm{~mm}$ ) and rolled like tube shape and then it is inserted in the interior surface of the photoreactor. Gas phase oxidation of toluene was tested on photo annular reactor, which is shown in Fig. 1.

The concentration of toluene was analyzed by gas chromatograph with a flame ionization detector. The gas samples were collected periodically using plastic syringe with suitable interval of time $(500 \mu \mathrm{L})$. The gas chromatograph oven temperature was held at $378 \mathrm{~K}$ and the detector temperature maintained constant at $523 \mathrm{~K}$.

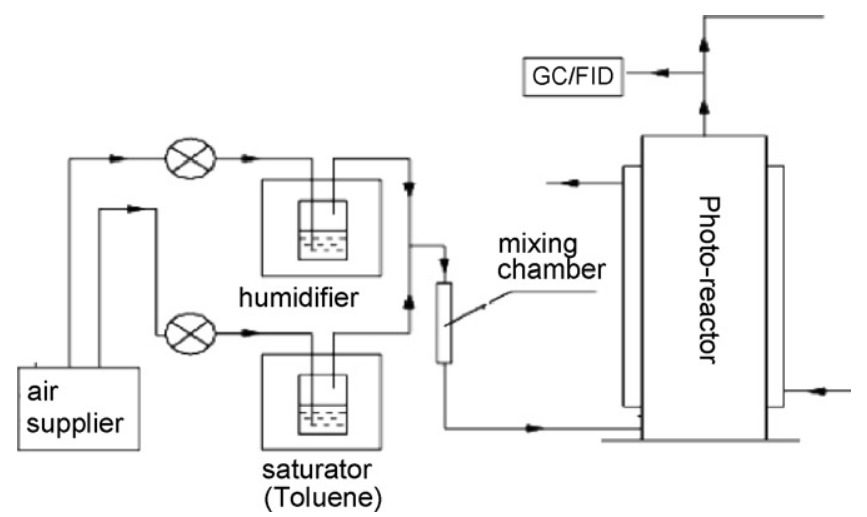

Fig. 1. Schematic representation of photocatalytic reactor.

The conversion $C(\%)$ of toluene was calculated as follows:

$C=\frac{\left(C_{\mathrm{i}}-C_{\mathrm{o}}\right) \times 100}{C_{\mathrm{i}}}$

where $C_{\mathrm{i}}$ is the inlet concentration and $C_{\mathrm{o}}$ is the outlet concentration at steady state.

The gas phase oxidation of toluene was tested on both pure titania (bulk) and manganese oxide doped titania materials. All catalysts tested up to $100 \mathrm{~min}$ after attain the equilibration and every $10 \mathrm{~min}$, concentration of toluene in the outlet were analyzed by gas chromatography using FID detector at $473 \mathrm{~K}$ and high pure nitrogen as used as carrier gas. Toluene samples collected from the tightly connected outlet by using $2 \mathrm{~mL}$ plastic syringe.

\subsection{Kinetic studies}

The kinetic and empirical equations, including the zero, first, Elovich and second order rate equations were applied to the toluene removal efficiency with respect to time. Linear forms of kinetic equations are derived by kinetic model reactions. The linear forms of different kinetic equations were applied to the adsorption data and their goodness of fit was evaluated based on the $R^{2}$ and standard error (S.E.).

- Zero order

$$
[\mathrm{A}]=[\mathrm{A}]_{\mathrm{o}}-k t
$$

- First order

$$
\ln [\mathrm{A}]=\ln [\mathrm{A}]_{\mathrm{o}}-k t
$$

- Second order

$$
\frac{1}{[\mathrm{~A}]}=\frac{1}{[\mathrm{~A}]_{\mathrm{o}}}+k t
$$

- Elovich

$$
q_{t}=\left(\frac{1}{\beta}\right) \ln \left(\frac{\alpha}{\beta}\right)+\left(\frac{1}{\beta}\right) \ln t
$$

where $q_{t}$ is amount of sorbate per unit mass of sorbent at time $t$. $\alpha$ and $\beta$ are empirical constants. Slope and intercept are $1 / \beta$ and $(1 / \beta) \ln (\alpha / \beta)$, respectively. 


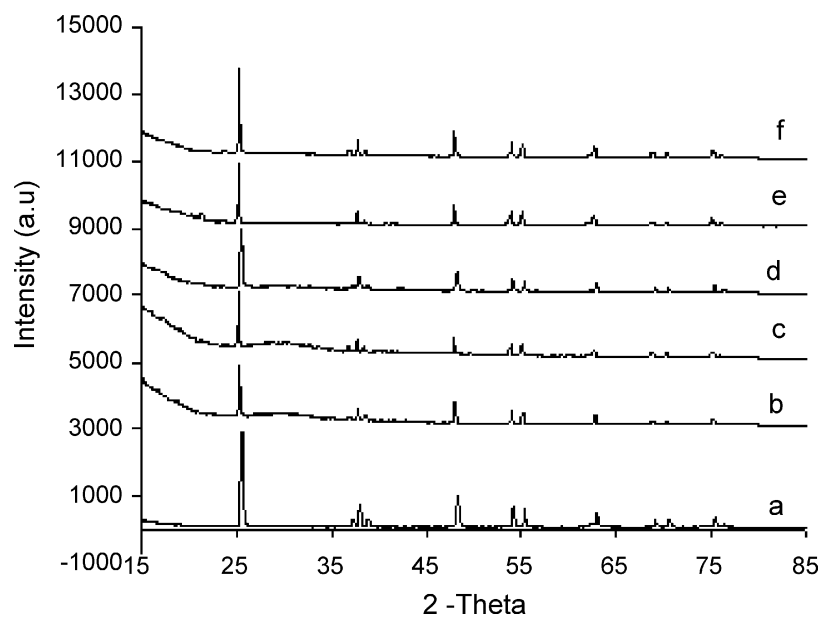

Fig. 2. X-ray diffraction patterns of manganese oxide OMS-titania material (a) $\mathrm{TiO}_{2}$, (b) $5 \mathrm{wt} \% \mathrm{KOL} / \mathrm{TiO}_{2}$, (c) $10 \mathrm{wt} \% \mathrm{~K}-\mathrm{OMS}-2 / \mathrm{TiO}_{2}$, (d) $15 \mathrm{wt} \% \mathrm{~K}$ $\mathrm{OMS} / \mathrm{TiO}_{2}$, (e) $10 \mathrm{wt} \% \mathrm{~K}-\mathrm{OMS} / \mathrm{TiO}_{2}$ (W), and (f) $5 \mathrm{wt} \% \mathrm{MnSO}_{4} / \mathrm{TiO}_{2}$.

\section{Results and discussion}

\subsection{X-ray diffraction study}

$\mathrm{X}$-ray diffraction pattern of different weight percent K-OL1 and K-OMS-2 type porous manganese oxide doped titania materials are shown in Fig. 2. All samples are showing the major phase of anatase with some amount of rutile phase. The major peak at $25.3^{\circ} 2 \theta, d$-spacing of $3.52 \AA$ ( 100$)$ of anatase is observed. It may be over imposed by presence of higher amount of manganese dopant. Gallardo-Amores et al. [14] reported the photocatalytic oxidation of 2-propanol on lower amount of manganese ion doped titanium dioxide. They observed the mixed phases for manganese doped titanium dioxide patterns in XRD, such as brookite and anatase phase. Manganese oxide was found to be well dispersed on the support surface as $\mathrm{Mn}^{3+}$ species, while part of it is stabilized into the $\mathrm{TiO}_{2}$ bulk as $\mathrm{Mn}^{4+}$ species [14]. This cation seems to hinder the support sintering and to favour the anatase to rutile phase transition at higher temperatures more than $973 \mathrm{~K}$. Doping of higher amount of manganese content in titanium dioxide such as up to $15 \mathrm{wt} \%$ loading may not cause the major partial phase transformation of anatase phase.

\subsection{Thermal and textural characterization of manganese oxide doped $\mathrm{TiO}_{2}$}

The thermal stability of as prepared material were tested by TG analysis (data not shown), all the manganese oxide OMS doped titania samples show the first weight loss or major weight loss observed between 723 and $973 \mathrm{~K}$, which suggest that manganese oxide OMS doped titania material are thermally stable until the temperature reaches $723 \mathrm{~K}\left(450^{\circ} \mathrm{C}\right)$. The TEM-EDAX pictures of $10 \mathrm{wt} \% \mathrm{~K}-\mathrm{OMS}-2$ (W) is shown in Fig. 3A, manganese oxide particles aggregated strongly and it dispersed on spherical morphology of titania surface. Spherical and thin plate morphology of titania particles clearly visible at higher resolution (Fig. 3A). The titania particle sizes range observed between 100 and $120 \mathrm{~nm}$, the lower magnification picture of manganese oxide doped titania shown in Fig. 3B, it clearly indicates the aggregated particle morphology of titanium dioxide and the corresponding TEM-EDAX spectrum shows the 0.4 at. $\% \mathrm{Mn}$ and 81 at. $\%$ for Ti. About $5 \mathrm{wt} \%$ manganese sulfate doped titanium dioxide shows the decrease in surface area $\left(19.8 \mathrm{~m}^{2} / \mathrm{g}\right) \mathrm{com}$ pared bulk $\mathrm{TiO}_{2}$ Nacalai Tesque $\left(36 \mathrm{~m}^{2} / \mathrm{g}\right)$ and its corresponding particle size is $0.443 \mu \mathrm{m}$ (mean value). Impregnation of manganese salt on titania surface some how inhibits the sintering of the titania surface results in decrease in the surface area of as synthesized catalyst ( $5 \mathrm{wt} \% \mathrm{MnSO}_{4} / \mathrm{TiO}_{2}$ ).

In the case of manganese oxide OMS doped $\mathrm{TiO}_{2}$ show the little higher in surface area value then bulk $\mathrm{TiO}_{2}$ due to catalyst preparation method and porosity of the dopents. About $5 \mathrm{wt} \%$ KOL doped $\mathrm{TiO}_{2}$ shows the surface area of $37 \mathrm{~m}^{2} / \mathrm{g}$ and $5 \mathrm{wt} \%$ K-OMS-2 doped $\mathrm{TiO}_{2}$ shows the $41 \mathrm{~m}^{2} / \mathrm{g}, 10 \mathrm{wt} \% \mathrm{~K}-\mathrm{OMS}-2$ doped $\mathrm{TiO}_{2}$ shows the $44 \mathrm{~m}^{2} / \mathrm{g}$ and $15 \mathrm{wt} \% \mathrm{~K}$-OMS-2 doped $\mathrm{TiO}_{2}$ shows the little decreased surface area such as $38 \mathrm{~m}^{2} / \mathrm{g}$, due to overloading of dopant.

\subsection{Catalytic test: gas phase oxidation of toluene on as synthesized materials}

Table 1 and Fig. 4 show the inlet concentration of reactant (toluene), catalyst loading amount, and irradiation time and removal efficiency percentage. About 5, 10 and $15 \mathrm{wt} \% \mathrm{~K}-$ OMS-2 coated titania catalysts, which is prepared by acetone medium and it show the total removal efficiency of toluene is
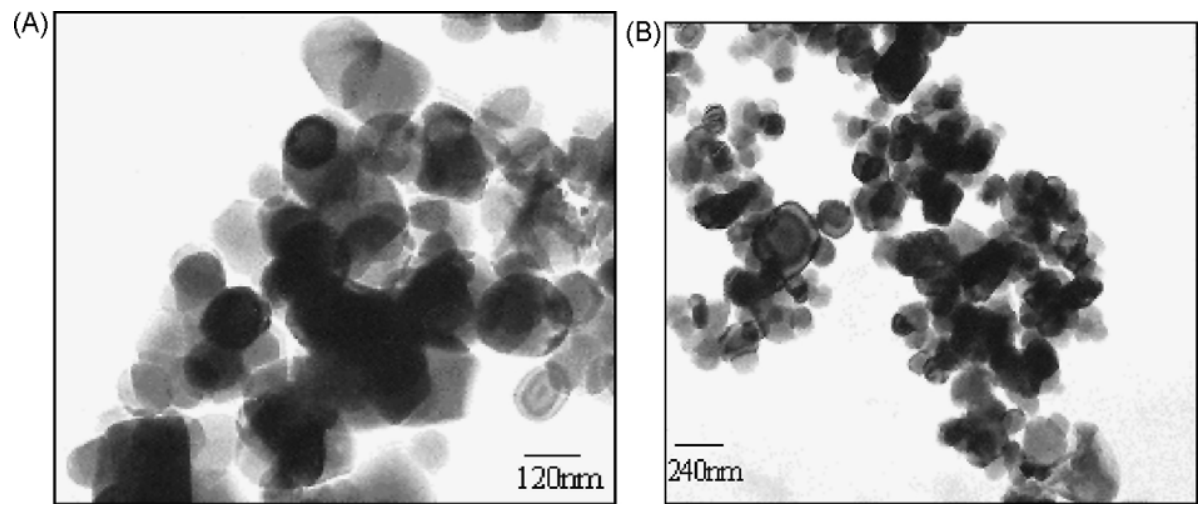

Fig. 3. Transmission electron micrographs of $10 \mathrm{wt} \% \mathrm{~K}-\mathrm{OMS}-2(\mathrm{~W})$-titania catalyst (A) $16,000 \times$ and (B) $8000 \times$ magnification. 
Table 1

Catalysts loading amount, inlet concentration, removal efficiency of toluene on manganese oxide OMS doped titania catalysts

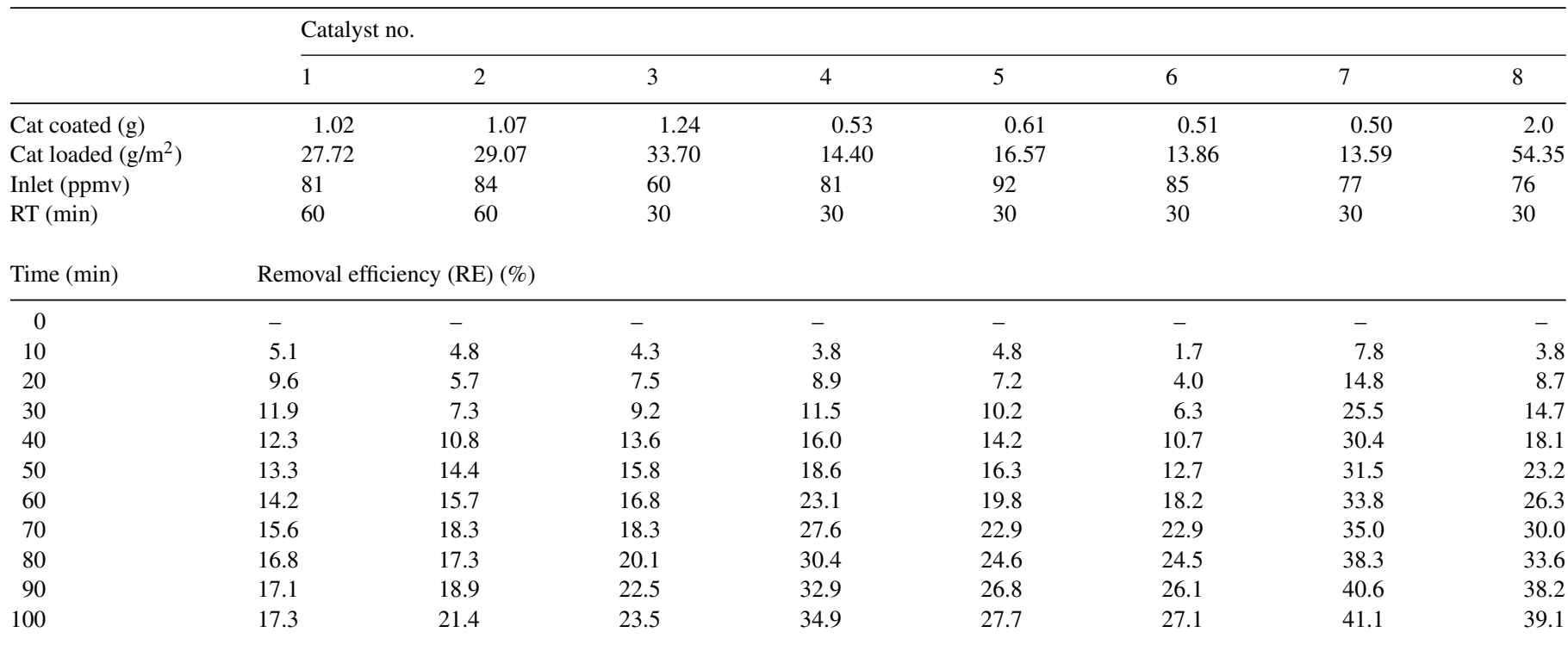

1, 5 wt $\%$ K-OMS-2; 2, 10 wt $\%$ K-OMS-2; 3, 15 wt $\%$ K-OMS-2; 4, 5 wt $\%$ KOL; 5,10 wt $\%$ KOL; 6, 5 wt $\%$ MnSO $_{4} ;$ 7, 10 wt $\%$ K-OMS-2 (W); 8 , TiO 2.

$23.5 \%$, where as $10 \mathrm{wt} \% \mathrm{~K}-\mathrm{OMS}-2(\mathrm{~W})$-titania materials prepared in water medium shows the good conversion nearly $41 \%$ compared to all other catalysts, in the case of commercial bulk titanium dioxide shows the $39 \%$ removal efficiency for toluene with higher catalysts loading $(2.0 \mathrm{~g})$ compared to manganese oxide doped titania catalyst loading $(0.5 \mathrm{~g})$. In term of catalysts laoded surface area $\left(\mathrm{g} / \mathrm{m}^{2}\right), 10 \mathrm{wt} \% \mathrm{~K}-\mathrm{OMS}-2 \mathrm{~W}$-doped titania, which shows the lower surface area $\left(13.59 \mathrm{~g} / \mathrm{m}^{2}\right)$ but it shows the good removal efficiency for toluene in photocatalytic reaction compared to bulk titania $\left(54.59 \mathrm{~g} / \mathrm{m}^{2}\right)$. The preparation method on manganese oxide doped titania have also been played the role in the degradation of toluene. Manganese oxide doped titania (prepared in water medium) shows the good conversion for toluene degradation compared to all other catalysts, which is due to adsorbed water molecules on manganese oxide doped titania, and it will cause the subsequent deprotanation in the reaction pathway.

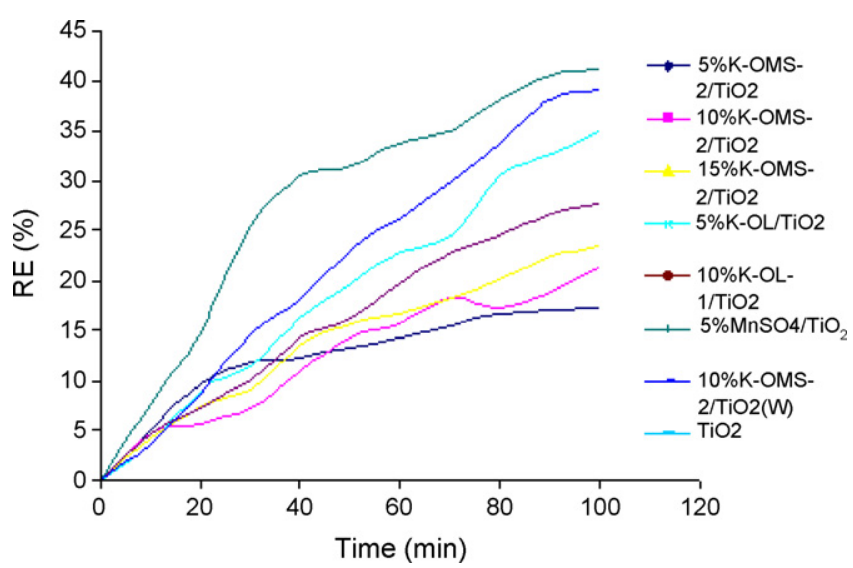

Fig. 4. Gas phase photocatalytic degradation of toluene on different manganese oxide doped titania catalysts.
Toluene degradation behavior on birnessite and cryptomelane doped titania (prepared in non-aqueous medium) show the different behavior in the photocatalytic reaction. About 5 and $10 \mathrm{wt} \%$ KOL doped titania catalysts show the better conversion 24-35\% compared to 5, 10 and $15 \mathrm{wt} \% \mathrm{~K}-\mathrm{OMS}-2$ doped titania catalysts $(17-23 \%)$ at the end of reaction (after $80 \mathrm{~min}$ ). Manganese sulfate doped titania a catalyst shows the better conversion for toluene degradation compared to K-OMS2 doped titania. It is because surface acid and base properties of the as synthesized catalysts may cause the difference in the toluene degradation ability, K-OMS-2 doped titania catalysts are hydrophobic in nature but in the case of birnessite doped titania catalysts have the basic site on its surface and probably it will enhace the toluene degradation. In the case of cryptomelane doped titania prepared in water shows the best formation for toluene degradation compared to all other catalysts including bulk titania. Cryptomelane doped titania prepared in water medium [K-OMS-2 (W)] shows the better removal efficiency from beginning of the reaction itself, i.e. after $20 \mathrm{~min}$ of the reaction it shows the $25.5 \%$ removal efficiency and it goes on increase up to $100 \mathrm{~min}$, it shows the longer steady state compared to bulk titania catalyst. Bulk titania catalyst show the $26 \%$ removal efficiency after $60 \mathrm{~min}$ of the photocatalytic degradation of toluene. K-OMS-2 (W) shows the total removal efficiency for toluene degradation around $41 \%$.

D'Hennezela et al. [29], studied the role of hydroxyl radical $\left({ }^{\bullet} \mathrm{OH}\right)$ formation on $\mathrm{TiO}_{2} / \mathrm{H}_{2} \mathrm{O}$ catalysts for benzene and toluene degradation and they observed the presence of $\mathrm{OH}$ radical (from adsorbed water on titania surface) and $\mathrm{Cl}$ radical (in the case of $\mathrm{HCl}$ treated $\mathrm{TiO}_{2}$ ) enhance the intermediate formation and finally facilitate the removal efficiency of the reactant. In the same way, water medium prepared manganese oxide OMS doped titania catalyst shows the good conversion for toluene degradation by $\mathrm{OH}$ radical ion formation ion the catalyst sur- 
(A)
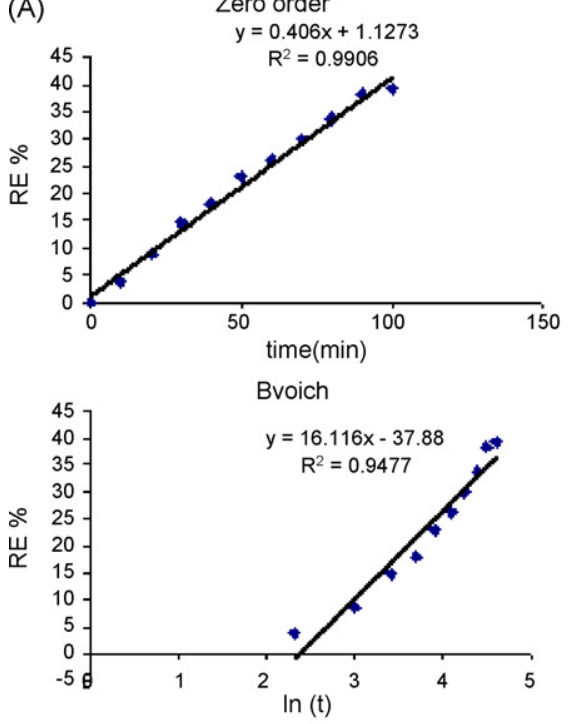

(B)
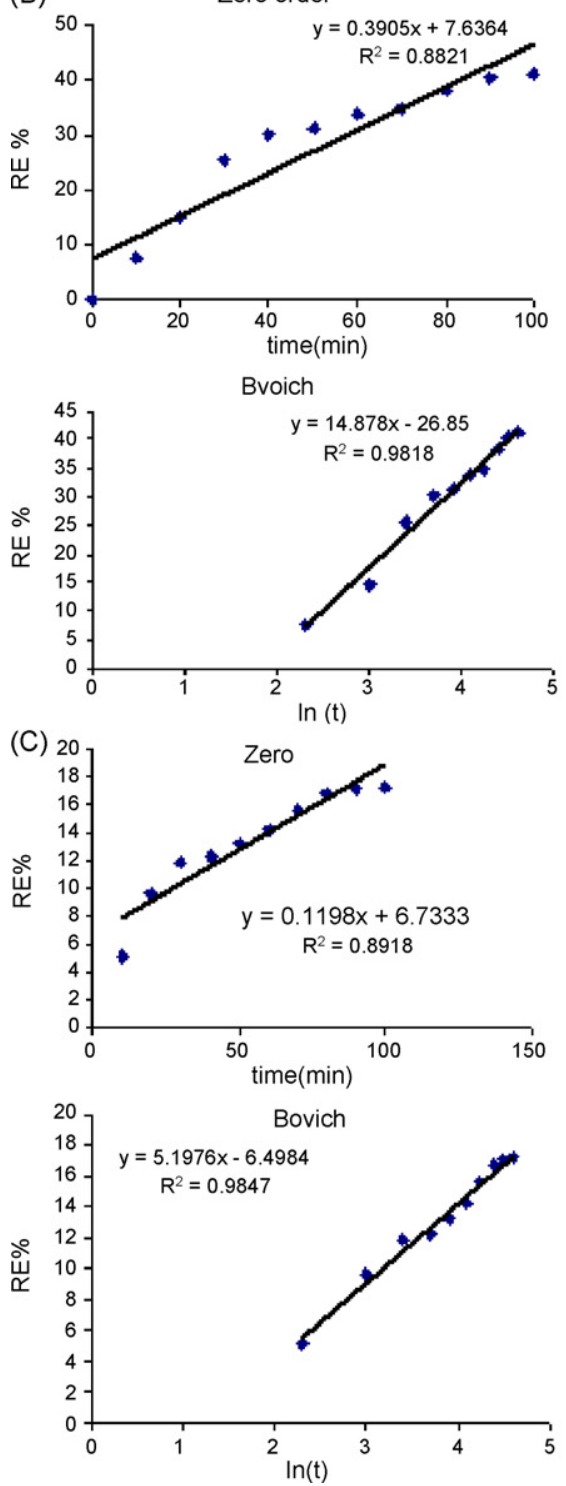

First order

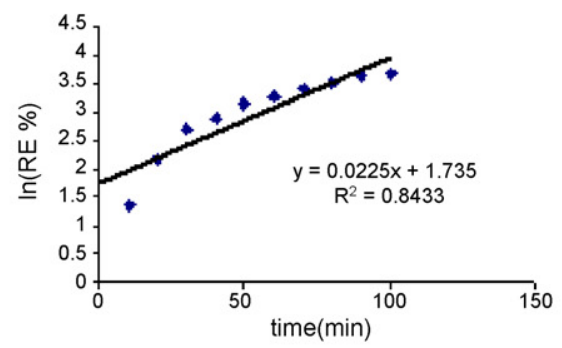

Second order

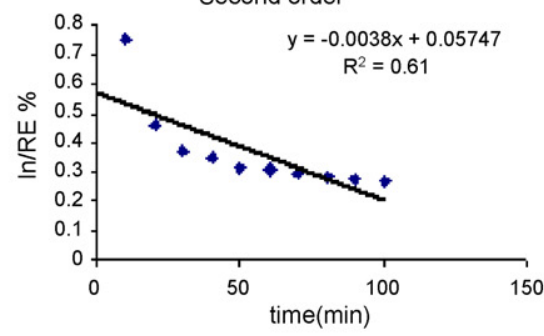

First order

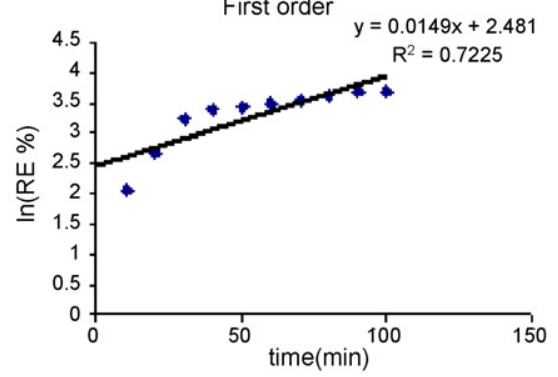

Second order
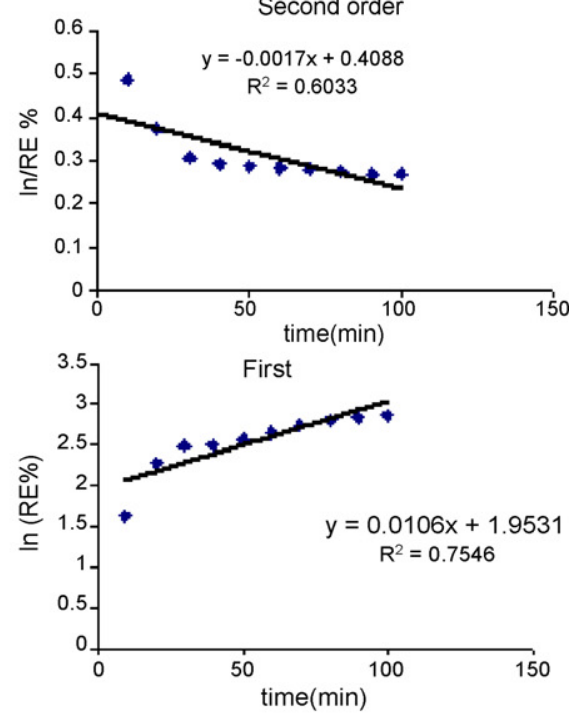

Second

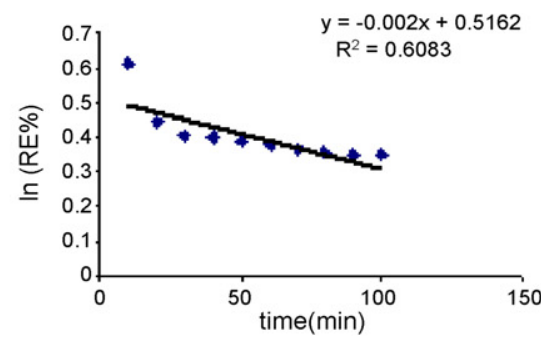

Fig. 5. Kinetic models of different manganese oxide doped titania catalysts of (A) 5 wt $\%$ K-OMS-2-titania, (B) 10 wt $\%$ K-OMS-2 (W)-titania, and (C) TiO 2 . 
face. The regeneration of the manganese oxide doped titania has also been studied and the used catalyst shows same performance after treated with water followed by calcinations in air. Therefore, the presence of adsorbed water or water medium prepared manganese oxide doped titania materials can provide the higher removal efficiency for degradation of toluene compared alcoholic route prepared catalysts and bulk titanium dioxide. In the present study, we used higher inlet concentration of toluene [77-92 ppmv] (Table 1) compared to other reports. Pengyi et al. [30], were studied the toluene degradation in the inlet concentration range of 5-20 ppmv and the steady state is same like our research data. This suggest that as prepared catalysts (manganese oxide doped titania) was attained the stable steady state and liner removal efficiency even at higher amount of inlet concentration.

\subsection{Kinetic studies}

Kinetic models of $5 \mathrm{wt} \% \mathrm{~K}-\mathrm{OMS}-2$ doped titania, $10 \mathrm{wt} \% \mathrm{~K}-$ OMS-2 (W)-titania and $\mathrm{TiO}_{2}$ was chosen to describe the kinetics of reaction and are shown in Fig. 5A-C. The goodness of fit was evaluated based on $R^{2}$ value. Based on our kinetic data, Elovich kinetic and zero order models are showing the highest $R^{2}$ value compared to other kinetic reaction models such as first and second order rate equations. Based on $R^{2}$-value the kinetic reaction models follow the following order for toluene removal efficiency

\section{Elovich $>$ Zero order $>$ First order $>$ Second order}

$\alpha$ and $\beta$ values from the Elovich equation has also found for catalysts such as $5 \mathrm{wt} \% \mathrm{~K}$-OMS-2-titania, $10 \mathrm{wt} \% \mathrm{~K}-\mathrm{OMS}-2$ (W)-titania, $\mathrm{TiO}_{2}$ (Fig. 5A-C). $\alpha$ values of the above catalysts are $0.929,0.895,0.863\left(\mathrm{ppmv}\right.$ as tolune $\mathrm{min}^{-1}$ ) and $\beta$ values are $0.192,0.672,0.0621\left((\mathrm{ppmv} \text { tolune } / 1 \mathrm{~g})^{-1}\right)$, respectively. Higher $\alpha$ with lower $\beta$ values indicates increase in the rate of reaction (based on Elvich reaction). The highest $R^{2}$-value (i.e., $R^{2} \geqq 0.9477$ ) observed for Elovich linear fit reaction (Fig. 5C). The high $R^{2}$ value (i.e., $\geqq 0.8821$ ), and the corresponding standard error (S.E.) was calculated from zero order linear fit such as, S.E. $=2.24$ for $5 \mathrm{wt} \%$ K-OMS-2-titania, S.E. $=4.99$ for $10 \mathrm{wt} \%$ K-OMS-2 (W)-titania, S.E. $=1.38$ for $\mathrm{TiO}_{2}$.

\section{Conclusions}

The porous manganese oxide doped titania materials were successfully prepared in aqueous and non-aqueous by adopting suitable synthetic procedure. The aggregated particle of manganese oxide (particle size in the range of $100-120 \mathrm{~nm}$ ) was dispersed on spherical and platy type morphology of titania. The synthesized samples show the good thermal stability and regeneration activity in the catalytic test. Manganese oxide OMS material doped titania show the improved surface area then bulk titanium dioxide. Manganese oxide doped titania prepared in aqueous medium (10 wt\% K-OMS-2 (W)) show the good conversion for toluene $(41 \%)$ with lower catalyst loading $(0.5 \mathrm{~g})$ compared to bulk titania, which shows the same conversion at higher catalyst loading amount $(2.0 \mathrm{~g})$. Birnessite type man- ganese oxide such as K-OL-1 manganese oxide doped titania catalyst shows the faster removal efficiency for toluene degradation compared cryptomelane K-OMS-2 doped titania and bulk titania catalysts with lower total removal efficiency at $100 \mathrm{~min}$. Kinetic models are shown the good linear fitness with the highest $R^{2}$ value for zero order and Elovich kinetic models. The above observations suggest that the low amount of manganese oxide KOMS-2 doped titania catalyst coating is sufficient to achieve the better conversion for volatile organic compounds degradation. It can reduce the catalyst synthesis cast compared to commercially available bulk titanium dioxide.

\section{Acknowledgments}

The authors express the sincere thanks to Mr. Jagan, Department of Chemical Engineering, IIT Madras, India, help to carry out the photocatalytic tests and also greatly thankful to National Science Council of Taiwan (NSC-95-2811-Z-002-005) for financial support. The authors also thank Dr. Po Neng Chang and Mr. Yue Min Chen for their help.

\section{References}

[1] S.C. Wilson, V. Burnett, K.S. Waterhouse, K.C. Jones, Volatile organic compounds in digested United Kingdom sewage sludges, Environ. Sci. Technol. 28 (1994) 259-266.

[2] J. Shah, H.B. Singh, Distribution of volatile organic chemicals in outdoor and indoor air: a national VOCs data base, Environ. Sci. Technol. 22 (1988) 1381-1388.

[3] C.F. Wilkinson, Cancer Risk Assessment. 1. Being more realistic about chemical carcinogenesis, Environ. Sci. Technol. 21 (1987) 843-847.

[4] L.A. Dibble, G.B. Raupp, Kinetics of the gas-solid heterogeneous photocatalytic oxidation of trichloroethylene by near UV illuminated titanium dioxide, Catal. Lett. 4 (1990) 345-354.

[5] J. Peral, D.F. Ollis, Heterogeneous photocatalytic oxidation of gas-phase organics for air purification: acetone, 1-butanol, butyraldehyde, formaldehyde, and $m$-xylene oxidation, J. Catal. 136 (1992) 554-565.

[6] D.F. Ollis, H. Al-Ekabi, Photocatalytic Purification and Treatment of Water and Air, Elsevier, Amsterdam, 1993.

[7] T.T. Shen, C.E. Schmidt, T.R. Card, Assessment and Control of VOC Emission from Waste Water Treatment and Disposal Facilities, Van Nostrand Reinhold, New York, 1993.

[8] R. Arroyo, G. Cordoba, J. Padilla, V.H. Lara, Influence of manganese ions on the anatase-rutile phase transition of $\mathrm{TiO}_{2}$ prepared by the sol-gel process, Mater. Lett. 54 (2002) 397-402.

[9] G.K. Boreskov, in: J.R. Anderson, M. Boudart (Eds.), Catalysis Science and Technology, vol. 3, Springer, Berlin, 1982, p. 39.

[10] Y.I. Pyatnitskii, Heterogeneous catalytic oxidation of aromatic hydrocarbons in the gas phase, Russ. Chem. Rev. 45 (1976) 762-776.

[11] X.Y. Jiang, Y.R. Jia, P.H. Huang, X.M. Zheng, Effect of pretreatment atmosphere on $\mathrm{CuO} / \mathrm{TiO}_{2}$ activities in $\mathrm{NO}+\mathrm{CO}$ reaction, Chem. Mater. Sci. 104 (2005) 169-175.

[12] F. Kapteijn, L. Singoredjo, M. van Driel, A. Andreini, J.A. Moulijn, G. Ramis, G. Busca, Alumina-supported manganese oxide catalysts. II. Surface characterization and adsorption of ammonia and nitric oxide, J. Catal. 150 (1994) 105-116.

[13] M. Baldi, V.S. Escribano, J.M.G. Amores, F. Milella, G. Busca, Characterization of manganese and iron oxides as combustion catalysts for propane and propene, Appl. Catal. B 17 (1998) L175-L182.

[14] J.M. Gallardo-Amores, T. Armaroli, G. Ramis, E. Finocchio, G. Busca, A study of anatase-supported Mn oxide as catalysts for 2-propanol oxidation, Appl. Catal. B 22 (1999) 249-259.

[15] M. Schiavello (Ed.), Heterogeneous Photocatalysis, John Wiley \& Sons, New York, 1995. 
[16] N. Serpone, E. Pelizzetti (Eds.), Photocatalysis: Fundamentals and Applications, John Wiley \& Sons, New York, 1989.

[17] A. Fujishima, K. Hashimoto, T. Watanabe, $\mathrm{TiO}_{2}$ Photocatalysis: Fundamentals and Applications, BKC, Tokyo, 1999.

[18] A.L. Linsebigler, G. Lu, J.T. Yates Jr., Photocatalysis on $\mathrm{TiO}_{2}$ surfaces: principles, mechanisms, and selected results, Chem. Rev. 95 (1995) 735-758.

[19] T. Ibusuki, K. Takeuchi, Toluene oxidation on UV irradiated titanium dioxide with and without $\mathrm{O}_{2}, \mathrm{NO}_{2}$ or $\mathrm{H}_{2} \mathrm{O}$ at ambient temperature, Atmos. Environ. 20 (1986) 1711-1715.

[20] T.N. Obee, R.T. Brown, $\mathrm{TiO}_{2}$ photo catalysis for indoor air applications: effects of humidity and trace contaminant levels on the oxidation rates of formaldehyde, toluene, and 1,3-butadiene, Environ. Sci. Technol. 29 (1995) $1223-1231$.

[21] Y. Luo, D.F. Ollis, Heterogeneous photocatalytic oxidation of trichloroethylene and toluene mixtures in air: kinetic promotion and inhibition, time-dependent catalyst activity, J. Catal. 163 (1996) 1-11.

[22] H. Einaga, S. Futamura, T. Ibusuki, Heterogeneous photocatalytic oxidation of benzene, toluene, cyclohexene and cyclohexane in humidified air: comparison of decomposition behavior on photoirradiated $\mathrm{TiO}_{2}$ catalyst, Appl. Catal. B 38 (2002) 215-225.

[23] V. Augugliaro, S. Coluccia, V. Loddo, L. Marchese, G. Martra, L. Palmisano, M. Schiavello, Photocatalytic oxidation of gaseous toluene on anatase $\mathrm{TiO}_{2}$ catalyst: mechanistic aspects and FT-IR investigation, Appl. Catal. B 20 (1999) 15-27.
[24] L. Cao, Z. Gao, S.L. Suib, T.N. Obee, S.O. Hay, J.D. Freihaut, Photocatalytic oxidation of toluene on nanoscale $\mathrm{TiO}_{2}$ catalysts: studies of deactivation and regeneration, J. Catal. 196 (2000) 253-261.

[25] A. Navio, M. Garcia Gómez, M.A. Pradera Adrian, J. Fuentes Mota, Partial or complete heterogeneous photocatalytic oxidation of neat toluene and 4-picoline in liquid organic oxygenated dispersions containing pure or iron-doped titania photocatalysts, J. Mol. Catal. 104 (1996) 329339.

[26] R. Enriquez, P. Pichat, interactions of humic acid, quinoline, and $\mathrm{TiO}_{2}$ in water in relation to quinoline photocatalytic removal, Langmuir 17 (2001) 6132-6137.

[27] H. Tada, H. Matsui, F. Shiota, M. Nomura, S. Ito, M. Yoshihara, K. Esumi, Heterosupramolecular photocatalysis: oxidation of organic compounds in nanospaces between surfactant bilayers formed on $\mathrm{TiO}_{2}$, Chem. Commun. (2002) 1678-1679.

[28] R. Jothiramalingam, B. Viswanathan, T.K. Varadarajan, Preparation, characterization and catalytic properties of cerium incorporated porous manganese oxide OMS-2 catalysts, Catal. Commun. 6 (2005) 41-45.

[29] O. D'Hennezela, P. Pichata, D.F. Ollis, Benzene and toluene gas-phase photocatalytic degradation over $\mathrm{H}_{2} \mathrm{O}$ and $\mathrm{HCL}$ pretreated $\mathrm{TiO}_{2}$ : by-products and mechanisms, J. Photochem. Photobiol. A: Chem. 118 (1998) 197204.

[30] Z. Pengyi, L. Fuyan, Y. Gang, C. Qing, Z. Wanpeng, A comparative study on decomposition of gaseous toluene by $\mathrm{O}_{3} / \mathrm{UV}, \mathrm{TiO}_{2} / \mathrm{UV}$ and $\mathrm{O}_{3} / \mathrm{TiO}_{2} / \mathrm{UV}$, J. Photochem. Photobiol. A: Chem. 156 (2003) 189-194. 